\title{
DISPONIBILIDAD LÉXICA EN ESTUDIANTES DE PRIMERO BÁSICO
}

\author{
Milko Cepeda Guerra* \\ Maribel Granada Azcárraga** \\ María Pomes Correa***
}

\section{Resumen}

El presente artículo da cuenta de los resultados de una indagación acerca de los índices de disponibilidad léxica en alumnos de primero básico en un colegio particular subvencionado de la ciudad de Talca. Para ello se tomó una muestra de centros de interés extraídos del Proyecto Panhispánico, que servirían de base para evaluar en los niños el nivel de desarrollo de los campos semánticos esperados para su edad, además de considerar como variable independiente el género de los estudiantes. El análisis de los datos se realizó a través del procesamiento computacional para el cálculo de índices y frecuencias. Los resultados indican que el índice de disponibilidad léxica es mayor en las niñas que en los niños, mientras que los niños muestran un promedio superior en la cantidad de vocablos producidos. Implicancias de estos hallazgos para la enseñanza y su impacto en el desarrollo del lenguaje oral y escrito son expuestas en este escrito.

Palabras clave: Índice de disponibilidad léxica, vocabulario, enseñanza explícita.

\section{LeXICAl AVAILABILITY IN FIRST GRADE STUDENTS}

\begin{abstract}
The article presents the results of a research experience on the index of lexical availability in 1st grade students from a subsidized school in of Talca city. Centers of interest were selected from the Panhispánico Project to gather the required information. These centers are the standards for assessing children's level of semantic development according their age. In addition, students' gender was considered as an independent variable. Data analysis was conducted using a specialized software to calculate vocabulary indexes and frequencies. Findings indicate that the index of lexical availability is greater in girls than boys, while boys display in average a larger number of oral words produced. Implications of these results on instruction and their impact on language development are discussed in this writing.
\end{abstract}

Keywords: Index of lexical availability, vocabulary, explicit instruction.

Recibido: 26-09-2013

Aceptado: 10-10-2013

* Chileno, Doctor en Lingüística, académico Universidad Católica del Maule, Chile. mcepeda@ucm.cl

** Chilena, Doctora en Filosofía y Ciencias de la Educación, por la Universidad Complutense de Madrid, académica Universidad Católica del Maule, Chile. mgranada@ucm.cl

*** Chilena, Doctora en Filosofía (Ph.D) en Educación Especial, intervención temprana, Universidad de Oregón (USA), académica Universidad Católica del Maule, Chile.mppomes@ucm.cl 
El desarrollo del vocabulario en los niños es un proceso complejo y progresivo en el que influyen factores intrínsecos y también factores de carácter externo como son la estimulación de la familia, la enseñanza formal escolar y el contexto cultural en general. El bagaje léxico del niño va experimentando modificaciones en sus aspectos cuantitativos y cualitativos, es decir, este conjunto de palabras que el niño/a dispone irá aumentando en cantidad y también irá incrementándose en su complejidad fonológica y semántica. Este conjunto léxico que el niño presenta se constituye en un dispositivo con el que el niño cuenta para acceder de mejor manera al lenguaje oral y escrito. Por tanto, existe una relación directa entre la cantidad y calidad de palabras que un niño/a posee y los procesos asociados a la comprensión y conocimiento del mundo que lo rodea.

Para entender mejor el proceso de desarrollo resulta importante conocer cuáles son las palabras que los niños son capaces de expresar de manera más expedita, lo que se conoce como Índice de Disponibilidad Léxica. Tal como plantea Valencia (1997), el Índice de Disponibilidad Léxica (IDL) es un indicador que nos muestra cuáles son las palabras que un grupo de sujetos pueden evocar de manera más rápida para utilizarlas en determinados temas, en procesos comunicativos con otros interlocutores. Poder investigar acerca del léxico disponible resulta relevante por cuanto se trata de un indicador del desarrollo cognitivo de los sujetos y da cuenta de la posibilidad que éstos tendrán posteriormente para leer y escribir de manera efectiva.

Diversos estudios acerca de disponibilidad léxica consideran que un eje fundamental en el aprendizaje de las lenguas es el factor lexical, llegando a plantear que sin este componente es imposible pensar en procesos comunicativos eficientes. Para Palapanidi (2012), sin embargo, la adquisición léxica es un proceso complejo en el que se ponen en juego variables como el ambiente donde se desenvuelven los sujetos:

El lexicón mental, donde reside toda la información de la competencia léxica, está estrechamente relacionado con nuestro entorno y por eso está sujeto a un incesante cambio provocado por la adquisición interminable de nuevas experiencias e informaciones. De esta manera, la adquisición del léxico es un proceso largo e interminable, durante el cual el alumno 
paulatinamente va aprendiendo las diversas acepciones de una palabra o elemento léxico. (p. 69)

Siguiendo la línea de Palapanidi (2012) y tratándose este estudio de niños en edad escolar, resulta imprescindible relevar un enfoque didáctico de la enseñanza del componente léxico, pues ello permitirá posteriormente un avance sustantivo en actividades como la lectura y la escritura por parte de los niños. Por lo demás, autores como Allen (1984), Gairns y Redman (1986), Morgan y Rinvolucri (1986) y Nation (1990), por nombrar algunos, insisten en la necesidad de que la enseñanza para la adquisición del léxico sea sistemática y ordenada.

Por otra parte, Bartol (1997) plantea que el componente léxico no sólo es un eje relevante al momento de evaluar la adquisición de la lengua materna, sino también de las estrategias de planificación didáctica que los profesores deban ocupar para trabajar con sus alumnos.

La riqueza, el alcance y el control del vocabulario son parámetros importantes en la adquisición de la lengua y, por ello, de la evaluación del dominio de la lengua que tiene el alumno, y de la planificación del aprendizaje y de la enseñanza de lenguas. (p. 86)

En torno a la importancia del acceso al léxico en la capacidad de compresión lectora, Galloso (2004) plantea que uno de los aspectos fundamentales para que se realice una lectura eficiente es el caudal léxico de los sujetos, el que debe ser rico en matices, de forma tal que permita a éstos ampliar su capacidad para comprender y producir textos. En el mismo sentido, Lin (2012) indica que es necesario contar con mecanismos que permitan conocer las palabras disponibles que requieren las personas en contextos determinados, pues el conocimiento léxico es una competencia lingüística básica para el proceso de aprendizaje y para el proceso comunicativo.

Así mismo, contar con el conocimiento del léxico de niños en edad escolar, podría impactar positivamente en los procesos de enseñanza que se utilizan para optimizar los resultados de aprendizaje. Sin embargo, los resultados delSistema de Medición de la Calidad de la Educación (SIMCE, 2012) en comprensión lectora, en $2^{\circ}$ básico, son de 250 puntos, mientras que la región del Maule es de 249. Para el mismo año se observa una brecha de 53 puntos entre el grupo socioeconómico bajo y alto. Si se considera el 
tipo de establecimiento, la evidencia muestra que existe una diferencia de 43 puntos entre los establecimientos municipales y particulares pagados. En este sentido, los establecimientos municipales obtienen en promedio 239 puntos, mientras que los particulares subvencionados obtienen 253 puntos. Las instituciones particulares pagadas obtienen 282 puntos en promedio. Si se observa la medición poniendo atención en la variable sexo, la medición indica que las mujeres obtienen mejores resultados que los hombres, estableciéndose una diferencia de 10 puntos (Educar Chile, 2012).

Las palabras facilitan el procesamiento de la información y, con ello, la comprensión de lo que se lee (Anderson \& Freebody, 1981; Perfetti, 1985). Los resultados mostrados por el SIMCE reafirman que los estudiantes presentan una escasa compresión lectora, además, de un escaso manejo de vocabulario, lo que no permitiría acceder al significado de la palabra $y$, por ende, a comprender lo que leen.

La realidad nacional hace plantear cuestiones que son necesarias para un avance significativo en las mediciones académicas. En este sentido es preciso señalar que el vocabulario es un indicador de conocimiento general que influye en la eficacia del aprendizaje, ya que ayuda al procesamiento cognitivo, mejorando la velocidad lectora y la comprensión (Bruning, Schorow, Norby y Ronning, 2005).

A su vez, el conocer más palabras en un texto puede incrementar la probabilidad de que un estudiante pueda utilizar de mejor manera el contexto para inferir el significado de otras unidades léxicas desconocidas en el discurso leído (Shefelbine, 1990). Entonces, en la medida en que se cuenta con un mayor repertorio léxico más pistas se tiene para extraer el significado de aquellas palabras que no son conocidas. Según los expertos la comprensión lectora adecuada depende de conocer cerca del 90 a 95\% de las palabras en un texto, lo que permite al lector captar la idea principal del texto, y construir el significado de las palabras desconocidas (Hirsch, 1996 citado por Eyzaguirre y Fontairem, 2008).

El aprendizaje de una nueva palabra permite una mayor delimitación del espacio semántico de todas las unidades léxicas relacionadas, lo que influye en una mayor riqueza de lenguaje (Lockett \& Shore, 2003; Shore \& Kempe, 1999). Por tanto, adquiriendo un conocimiento profundo de este ámbito del lenguaje los estudiantes serían capaces de desarrollar el aprendizaje de otras palabras relacionadas, incluso si éstas no han sido 
consideradas dentro de su instrucción en el aula, lo que establece un carácter generativo del aprendizaje. Este conocimiento profundo de las palabras se logrará con mayor efectividad si este aprendizaje se planifica de forma estructurada y se presenta de manera sistemática y explícita.

Por su parte, Rosenshine (1987) describe la enseñanza explícita como un método sistemático, con énfasis en el procedimiento de pasos pequeños que van verificando la comprensión del aprendiz para asegurar experiencias exitosas durante el proceso de aprendizaje. En este sentido, una enseñanza explícita entregará ayudas ajustadas al estudiante, que van apoyando la progresión del aprendizaje. Un primer paso es la selección y secuencia lógica del contenido; luego, a través de la división en unidades pequeñas según las capacidades cognitivas de los estudiantes (memoria de trabajo, atención y conocimiento previo). La enseñanza se caracteriza por una clara descripción de habilidades, seguidas por una práctica guiada y retroalimentación oportuna. El profesor tiene una alta participación o apoyo directo al inicio de la enseñanza, la que se va retirando a medida que el estudiante experimenta experticia y autonomía en su desempeño.

Otras evidencias se suman al señalar que se hace muy poca instrucción explícita directa y sistemática antes de 3 básico (Baumman, Kame`Enui, \& Ash, 2003; National Reading Panel, 2000; Stahl \& Fairbanks, 1986). En relación a esto, diversas investigaciones señalan que no es recomendable dejar al azar el aprendizaje de nuevo vocabulario, enfatizando que las nuevas palabras se deben enseñar de manera explícita (Borrero, 2008). En este marco, Elleman (2009) enfatiza el hecho de que hay muy pocos estudios en el contexto escolar real que midan la comprensión y manejo de vocabulario.

Dentro del proceso de desarrollo de vocabulario se observan algunas diferencias entre hombres y mujeres. En ese contexto, posturas constructivistas y esencialistas delinean las razones que explicarían tales diferencias. La primera considera que el contexto social es un factor relevante, puesto que son los padres, la escuela y los pares los que condicionan las formas con que los menores van a adquirir y posteriormente usar el lenguaje. Gillani (2010) plantea que los factores sociales son determinantes en la adquisición y uso de la lengua materna y sus diferencias según género, entre estos factores se destacan la influencia de los padres, la escuela, la televisión y las prácticas socioculturales. 
Por su parte, los esencialistas tienden a apuntar a que existirían diferencias biológicas, como la organización del cerebro en hombres y mujeres y la maduración cognitiva, que permitirían, por ejemplo, una preeminencia sobre los hombres en la adquisición del lenguaje. Así, la explicación de Gillani (2010) indica:

las aproximaciones esencialistas sobre la adquisición del lenguaje hacen referencia a diferencias biológicas entre mujeres y hombres como la principal explicación de las diferencias de género en la adquisición de una primera lengua. En relación con esta idea, la explicación más recurrente ha sido la superioridad femenina en el aprendizaje de una lengua. (p. 3)

En el contexto nacional existe escasa información sobre el índice de disponibilidad léxica que los niños poseen en edad escolar y sobre la existencia de diferencias entre niños y niñas en cuanto a la cantidad y tipo de palabras que ellos evocan. Este desconocimiento impacta negativamente en la comprensión general que se tiene del desarrollo lingüístico de los niños chilenos y también en el abordaje pedagógico de la enseñanza y aprendizaje del lenguaje oral y escrito. En función de los antecedentes presentados, cabe señalar que es esencial conocer cuántas y cuáles son las palabras que disponen los niños y niñas al inicio de la etapa escolar, para poder potenciar la enseñanza y el incremento del léxico, con el propósito de mejorar la comprensión del lenguaje oral y escrito tan necesario para todos los aprendizajes. Por tanto, el propósito de este escrito es dar a conocer los resultados de una experiencia inicial que se propuso los siguientes objetivos: (a) determinar cuánto y cuál es el léxico disponible de niños y niñas de primer año básico de un colegio particular subvencionado de la ciudad de Talca; (b) comparar el léxico disponible de los niños y niñas evaluados. La hipótesis de este estudio plantea que existen diferencias en el índice de disponibilidad léxica, en el número de vocablos y promedio de palabras emitidas por los niños y niñas evaluados en un primer año básico.

\section{Método}

\section{Participantes}

La muestra para esta investigación está compuesta de 45 estudiantes ( $\mathrm{n}$ niños $=25 ; \mathrm{n}$ niñas $=20$ ) dentro de un universo de 62 alumnos. Los 
estudiantes pertenecen a un primer año básico de un colegio particular subvencionado de la ciudad de Talca, cuyas edades fluctúan entre los 6 y 7 años de edad. Como criterio de inclusión se consideró estudiantes cuya lengua materna fuera el español de Chile y que estuvieran en 1er año básico al momento de ser evaluados. Como criterio de exclusión se consideró la presencia de dificultades del lenguaje diagnosticadas, discapacidad intelectual $\mathbf{u}$ otras alteraciones o patologías asociadas al desarrollo. La siguiente figura indica las características de selección para la muestra:

Tabla 1

\section{Composición de la Muestra}

\begin{tabular}{lc}
\hline Características & $\mathrm{N} /$ porcentaje \\
\hline Tamaño poblacional & 63 \\
Confiabilidad & .95 \\
Error muestral & .05 \\
Potencia & .70 \\
Total participantes & 45 \\
\hline
\end{tabular}

Los alumnos evaluados pertenecen a un colegio particular subvencionado y mayoritariamente pertenecen a zonas urbanas de la ciudad de Talca, así como lo muestra la figura 1.

\section{Figura 1}

Lugar de residencia del grupo familiar de los niños y niñas evaluados

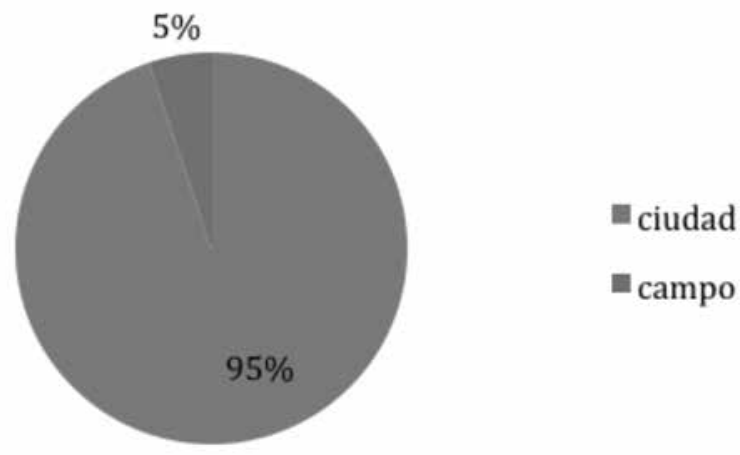


Además, se puede precisar que el colegio está catalogado en nivel socioeconómico medio, donde el ingreso más alto de los padres de los estudiantes evaluados está en el rango de 500 mil a 800 mil pesos, tal como se expone en la figura 2.

Figura 2

\section{Ingresos mensuales de las familias de los niños y niñas evaluados}

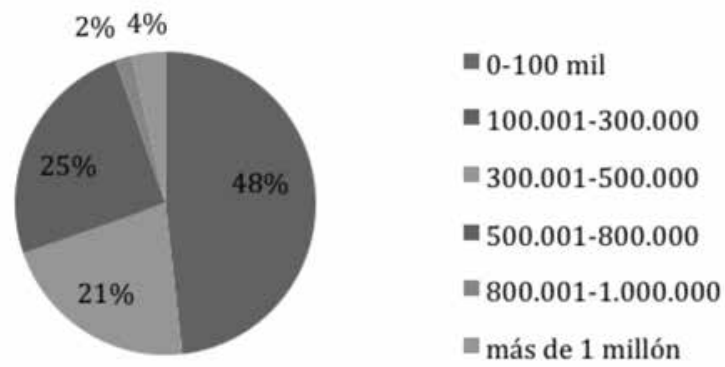

Otra información recogida y que caracteriza a la muestra es el tiempo que los estudiantes dedican en el día a ver televisión. El colegio tiene un régimen de "jornada completa", que implica un horario de entrada a las 8:30 horas y de salida a las 15:30 horas. En consideración al tiempo que los estudiantes tienen disponible para actividades extraescolares, se puede observar que sólo el $12 \%$ de ellos ve televisión menos de una hora al día, por tanto el $86 \%$ ve televisión entre 1 y 9 horas, tal como se observa en la Figura 3. Por otra parte, si se considera que en el país los canales más vistos por los niños y niñas como lo son los dibujos animados, utilizan español neutro, esto permite predecir la aparición en la muestra de algunas unidades léxicas no pertenecientes al habla de la variante dialectal del español de Chile. 
Figura 3

Número de horas que ven televisión y programas más vistos por los estudiantes
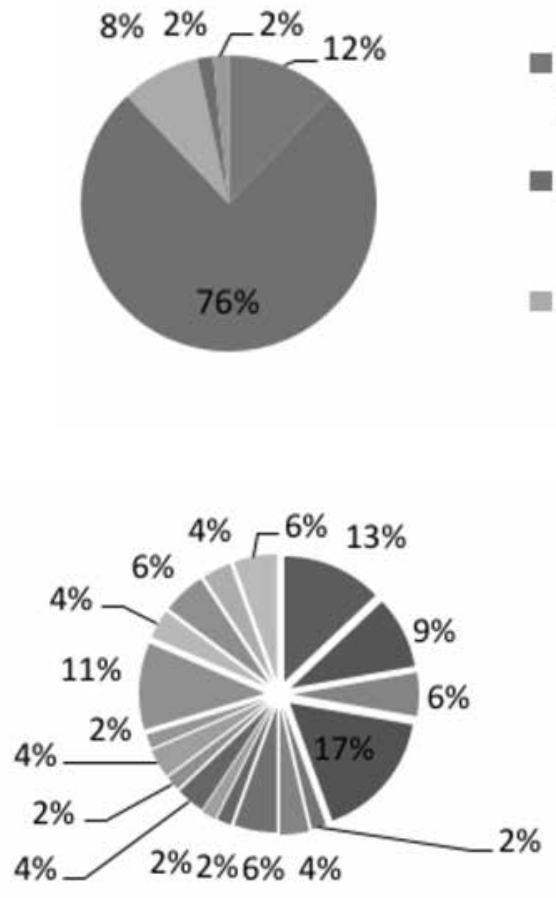

menos de una

hora

1-3 horas

4-6 horas

\section{Materiales}

Se seleccionaron once centros o categorías semánticas para ser utilizadas con los estudiantes. A partir de estos centros de interés se espera que los niños y niñas evoquen palabras que pertenezcan a ellos. Se han seleccionado estos centros de interés a partir del Proyecto Panhispánico, cuyo objetivo fue elaborar diccionarios de disponibilidad léxica para las diversas zonas del mundo hispánico. La homogeneidad de criterios permitiría establecer comparaciones de tipo lingüístico, etnográfico y cultural, dibujar áreas de difusión y, en general, servir de punto de partida para realizar análisis posteriores de disponibilidad léxica. Los campos seleccionados para este estudio son: "partes del cuerpo", "ropa y calzado", "alimentos y bebidas", "medios de transporte", "animales, juguetes", "ciudad", "casa", "profesiones", "escuela y colores". 


\section{Instrumentos}

La etapa de recogida de información requirió de dos instrumentos. El primero es un formulario de identificación, en el que los padres de los estudiantes debían aportar con datos diversos, como número de horas que los niños ven televisión, programas favoritos, escolaridad de los padres, actividad que desarrollan, lugar de residencia y rango de ingresos del núcleo familiar. El segundo mecanismo utilizado para conocer cuáles y cuántas son las palabras que disponen los niños y niñas de primer año básico, es un protocolo que incorpora los 11 centros de interés en un formato de cuestionario, donde los participantes disponían de dos minutos para entregar su léxico en cada uno de los once centros, ordenados de la siguiente manera: "partes del cuerpo", "ropa y calzado", "alimentos y bebidas", "medios de transporte", "animales", "juguetes", "ciudad", "casa", "profesiones", "escuela y colores". El ordenamiento de los centros de interés se realizó de esta manera para evitar que se produjera priming (e.i, asociación de ideas) en la respuesta de los estudiantes.

\section{Procedimientos}

Este estudio tiene un carácter cuantitativo, no experimental, descriptivo y considera como variable independiente el género de los participantes, siendo la variable dependiente el índice de disponibilidad léxica alcanzado.

Para llevar a cabo esta experiencia investigativa se desarrollaron las siguientes acciones. Primeramente se coordinó con la Dirección del establecimiento la factibilidad de realizar el estudio, los horarios y materiales a utilizar. Además se hizo llegar al colegio un consentimiento informado para que los padres decidieran sobre la participación de sus hijos en él. En un segundo momento se informó a los padres sobre el proyecto en una reunión de apoderados y se aplicó el formulario de identificación para recoger datos demográficos de las familias. Posteriormente, se capacitó a estudiantes de la carrera de Educación Especial para que aplicaran el cuestionario de centros de interés. La aplicación se realizó en una mañana en donde cada examinador evaluó individualmente a niños/as en un tiempo aproximado de 20 minutos, registrando de manera escrita y digital el léxico disponible de cada estudiante para luego ser transcrito. Esta información fue luego compilada para su posterior análisis. 
Para organizar y validar los datos recogidos de la aplicación del cuestionario de disponibilidad léxica, se tomaron las siguientes decisiones: (a) digitar todo en minúscula, (b) anotar en singular sustantivos y adjetivos, (c) remitir los diminutivos a la forma primitiva, (d) remitir todas las formas verbales al infinitivo, excepto gerundio y participio, (e) las palabras compuestas se digitaron unidas por guiones.

Para el procesamiento y análisis de los datos, el material se sometió a procesamiento computacional para el cálculo de índices y frecuencias. El más importante es el índice de disponibilidad. Para lo anterior se utilizó un programa computacional llamado Dispogen II (Echeverría et al., 2005). Esta es una aplicación hecha en MatLab, programa especializado en cálculos sobre la base de matrices y en el análisis estadístico de datos multivariantes, como es el caso de la disponibilidad léxica.

Dispogen II calcula los índices de disponibilidad léxica a través de la fórmula de Strassburguer y López-Chávez creada en 1987. Esta fórmula tiene la ventaja de trabajar con un exponente cuya función es asintótica a cero, es decir, nunca habrá un resultado cuyo valor sea cero. De este modo, los valores resultantes mantienen su capacidad discriminante.

Tres son los índices considerados en las últimas investigaciones (Urzúa, 2006) como los que más aportan a la determinación de la riqueza léxica de los sujetos. Ellos son el índice de cohesión (IC), el total de palabras diferentes $(\mathrm{PD})$ y el promedio de respuestas $(\mathrm{XR})$.

El índice de cohesión es un indicador del grado de coincidencia en las respuestas. Esto permite interpretar más adecuadamente los datos del índice anterior, pues el grado de homogeneidad en el uso del léxico está en proporción directa con el grado de disponibilidad que ese léxico tiene para todos los sujetos como conjunto. El total de palabras diferentes es un índice que da cuenta del total de vocablos conocidos por el grupo muestral. Por su parte, el promedio de respuestas indica cuántos son los vocablos que, en promedio, poseen los sujetos para su comunicación en el ámbito de conocimiento que explora el centro de interés respectivo.

\section{Resultados}

En términos generales y luego de realizar el análisis de los datos de los 11 centros de interés utilizados, se puede establecer que los índices de disponibilidad léxica de los estudiantes evaluados no sobrepasa el nivel 0.25 . 
El centro de interés que presenta el mayor nivel es el centro de interés de los "colores" pues es el que presenta un mayor grado de disponibilidad en el repertorio de palabras evidenciadas por los niños y niñas.

El centro de interés que presentó un nivel por sobre 0.1 es el "cuerpo humano", mientras que los demás centros de interés se encuentran por debajo del nivel 0.1. Por otro lado, los centros "juegos", "ciudad" y "profesiones y oficios" son los que presentaron una menor disponibilidad léxica al momento de ejecutar la evaluación.

Figura 4

Índice de disponibilidad léxica

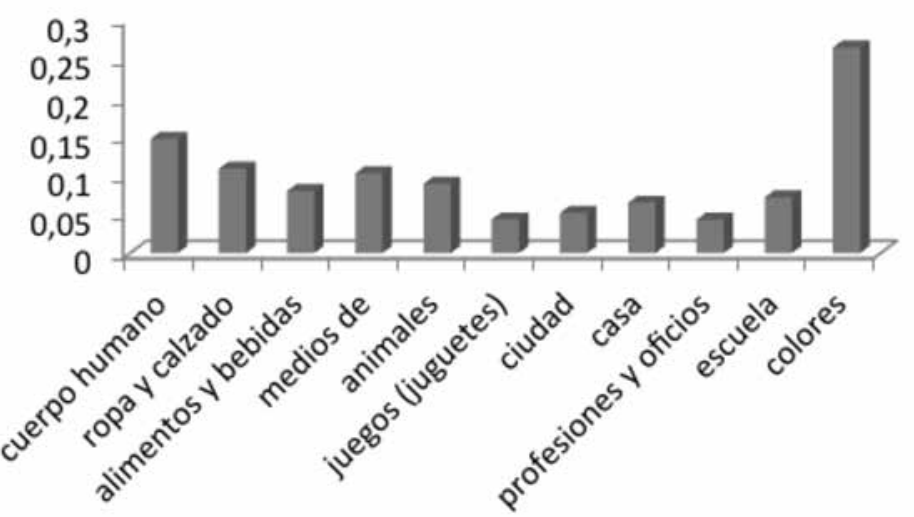

Si se considera el número de palabras diferentes evocadas por los estudiantes, podemos apreciar en la figura 5 que la población total de evaluados tuvo un mejor desempeño en los centros de interés de "bebidas y alimentos", "ciudad" y "casa" con 140 y 160 palabras, respectivamente; mientras que los menores desempeños estuvieron en "partes del cuerpo", "ropa y calzado", "medios de transportes" y "colores". Queda de manifiesto que aunque los informantes evoquen un número importantes de palabras distintas, esto no necesarimente se traduce en una mayor disponibilidad de estos repertorios en los sujetos. 
Figura 5

Número de vocablos evocados por los niños y niñas

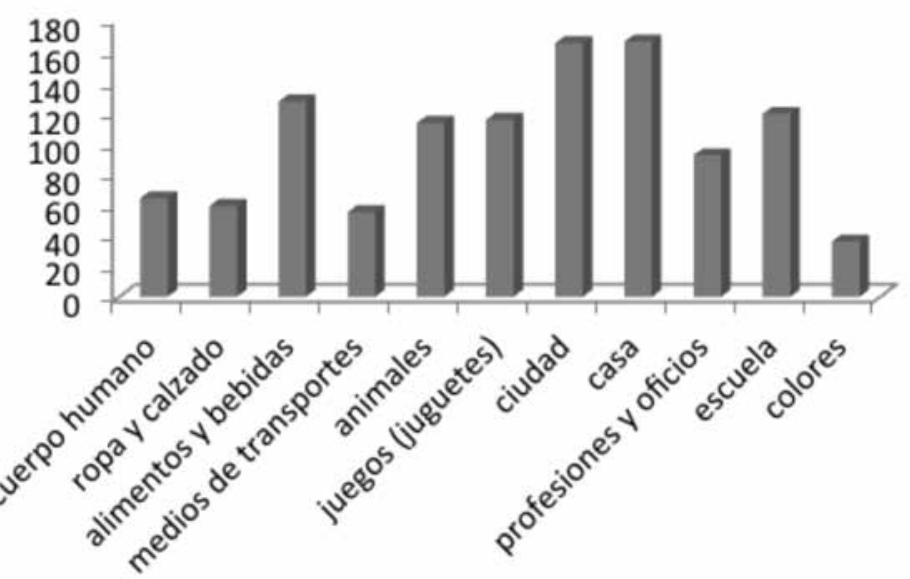

El promedio de palabras tal como lo presenta la figura 6, indica que los estudiantes logran evocar, en el mejor de los casos, 12 unidades. Se puede apreciar también que son más los centros de interes en que los niños y niñas tienen un mejor desempeño, con 7 campos por sobre la decena de palabras. Por otra parte, los centros de "medios de transportes", "juegos" y "profesiones y oficios" muestran promedios menores al momento de evocar unidades léxicas.

\section{Figura 6}

\section{Promedio de palabras evocadas por los niños y niñas}

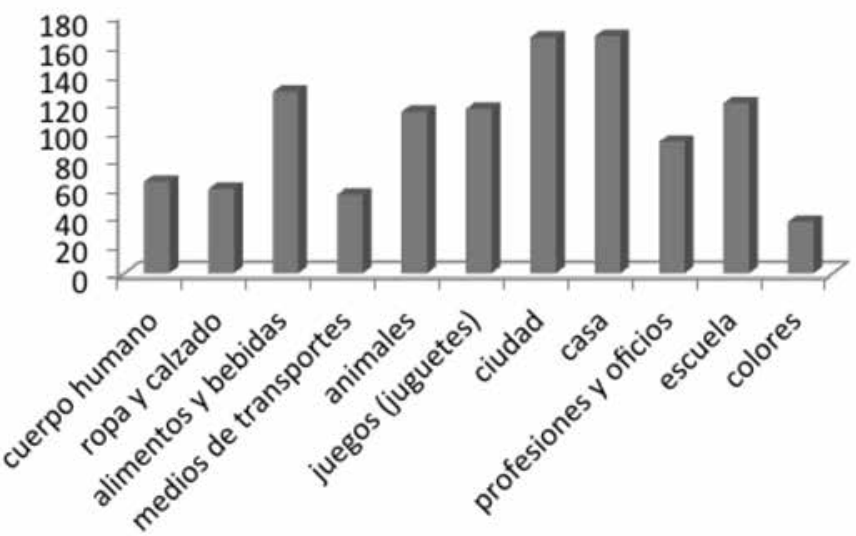


Si se realiza una comparación entre hombres y mujeres, como lo muestra la figura 7, podemos observar que los índices de disponibilidad léxica de las niñas es mayor al de los niños en todos los centros de interés, destacándose el caso de los colores donde ambos grupos puntuaron entre 0,4 y 0,5. Se puede observar también que los centros más conflictivos para los alumnos siguen siendo "juegos", "ciudad", "profesiones" y "casa", todos ellos menores o iguales a 0.1 .

\section{Figura 7}

\section{Comparación del índice de disponibilidad léxica entre niños y niñas}

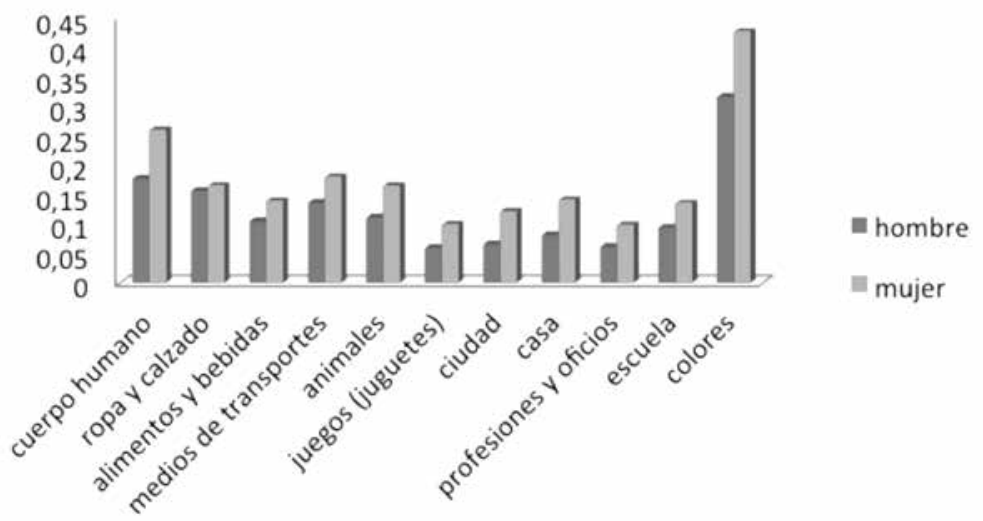

Si nos remitimos a la figura 8 , se puede establecer que el promedio de palabras evocadas, tanto por niños y niñas, es prácticamente similar, aún en los campos conflictivos mencionados anteriormentes. Sin embargo, cuando se compara el número de palabras distintas, los niños son capaces de nombrar un número mayor de unidades léxicas que las niñas, tal como queda representado en la figura 9. 
Figura 8

Comparación del promedio de palabras producidas por niños y niñas

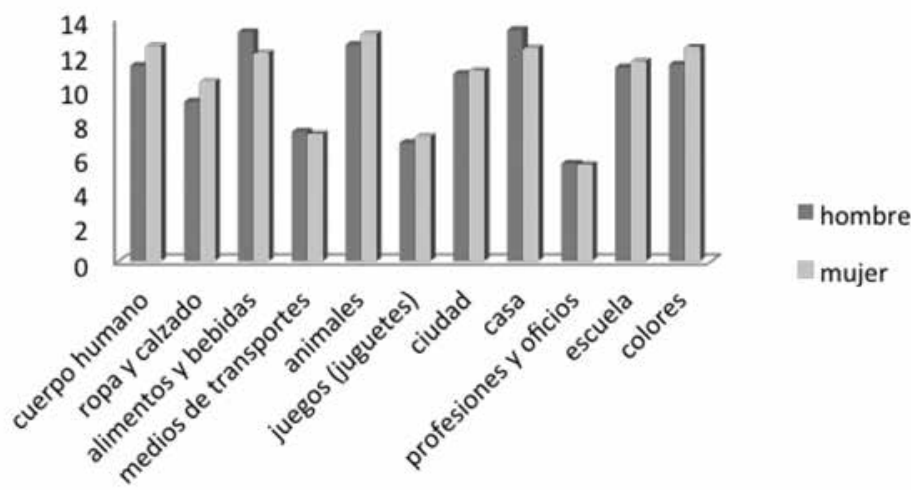

Figura 9

Comparación del número de vocablos entre niños y niñas

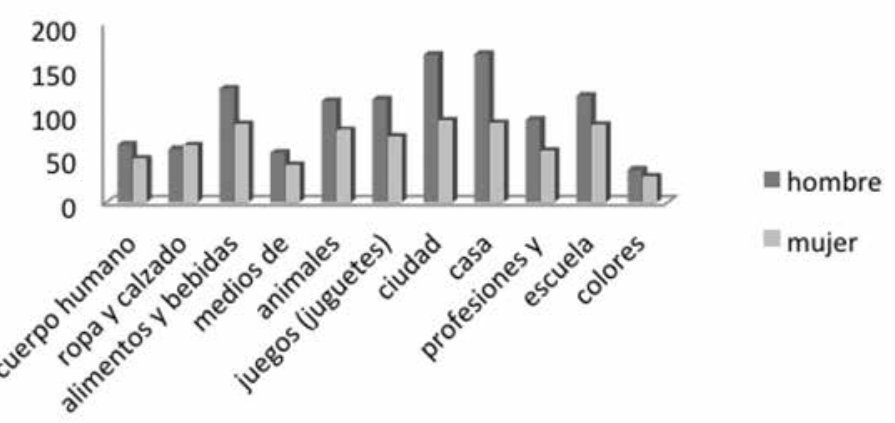

Es importante además analizar los resultados a partir de cada uno de los centros de interés. Si se observan los tipos de respuestas de los estudiantes de acuerdo al centro de interés "cuerpo humano", se puede advertir que ellos evocan palabras sin que éstas se constituyan en nodos semánticos. Por ejemplo, las unidades más disponibles corresponden a partes espacialmente distantes, tal como se observa en la tabla 2. 
En cuanto al campo "ropa y calzado" incluido en la tabla 2, las unidades más disponibles recogen prendas que son de uso más cotidiano, por ejemplo: polera, pantalón, calcetín, zapato, zapatilla, y que son parte del uniforme. No aparecen, por tanto, unidades léxicas que no formen parte de su vestimenta habitual de temporada, como botas, bufandas, etc.

En cuanto a las bebidas y alimentos, se aprecia la irrupción de unidades metonímicas (e.g, Coca-cola, Fanta, Sprite) como las más disponibles. En concreto, pareciera ser que los alumnos recuerdan más los nombres de las bebidas, mientras que los alimentos están relacionados con algunas verduras, el pan que es parte central de la dieta del país, además de arroz $\mathrm{y}$ tallarines.

El centro "medios de transportes" resulta ser conflictivo debido a que exhibe las unidades más dispersas de la muestra. Por otra parte, es un campo en el que los alumnos no presentan un buen desempeño en cuanto al número de unidades. Sólo la unidad "auto" tiene un alto índice de disponibilidad léxica, mientras que las demás son más bien dispersas. Así se visualiza en la tabla 2.

León, perro y gato son las palabras más diponibles del centro "animales", luego de las cuales aparecen elefante, jirafa y tigre. Al respecto, llama la atención que dentro de los mayores índices de disponibilidad léxica no aparezcan unidades léxicas que tengan relación con el entorno (Talca es una ciudad con los mayores índices de ruralidad de Chile). Sin embargo, los números exhibidos por este campo también son bastantes menores en la muestra, tal como se señala en la tabla 3.

El campo 6 también presenta elementos metonímicos. Más aún, en este centro irrumpen préstamos anglófonos producto de que algunos de los juguetes preferidos por los niños y niñas tienen que ver con series y personajes norteamericanos y que se reafirma día a día alentado por el merchandising de la industria juguetera. Dentro de los juegos llama la atención la "pillá", juego popular en la infancia, tal como se observa en la tabla 3 . 
Tabla 2

Índice de Disponibilidad Léxica para los Centros Cuerpo Humano, Ropa y Calzado, Bebidas y Alimentos y Medios de Transporte

\begin{tabular}{llllllll}
\hline \multicolumn{2}{c}{\begin{tabular}{c} 
Cuerpo \\
\multicolumn{2}{c}{ Humano }
\end{tabular}} & & \multicolumn{2}{c}{ Alimentos } & \multicolumn{2}{c}{ Transporte } \\
\hline Vocablo & IDL & Vocablo & IDL & Vocablo & IDL & Vocablo & IDL \\
Cabeza & 0.72 & Polera & 0.72 & Coca-cola & 0.58 & Auto & 0.84 \\
Mano & 0.59 & Pantalón & 0.65 & Fanta & 0.39 & Camión & 0.42 \\
Pie & 0.56 & Calcetín & 0.42 & Sprite & 0.33 & Avión & 0.32 \\
Brazo & 0.43 & Zapato & 0.37 & Lechuga & 0.26 & Bus & 0.26 \\
Ojo & 0.37 & Zapatilla & 0.26 & Manzana & 0.24 & Moto & 0.24 \\
Pierna & 0.35 & Short & 0.24 & Pan & 0.22 & Camioneta & 0.23 \\
Nariz & 0.27 & Chaleco & 0.20 & Poroto & 0.20 & Micro & 0.23 \\
Cuello & 0.24 & Vestido & 0.19 & Jugo & 0.18 & Bicicleta & 0.19 \\
Boca & 0.24 & Gorro & 0.16 & Tallarín & 0.18 & Bote & 0.17 \\
Rodilla & 0.24 & Buzo & 0.16 & Carne & 0.17 & Transporte & 0.14 \\
Dedo & 0.23 & Polerón & 0.14 & Arroz & 0.17 & Tren & 0.10 \\
Hombro & 0.20 & Camisa & 0.11 & Zanahoria & 0.16 & & - \\
Oreja & 0.17 & Chala & 0.10 & Papa-frita & 0.15 & & - \\
Codo & 0.14 & & - & Lenteja & 0.13 & & - \\
Muñeca & 0.12 & & - & Puré & 0.13 & & - \\
Hueso & 0.10 & & - & & - & & - \\
\hline
\end{tabular}


Tabla 3

Índice de Disponibilidad Léxica para los Centros Animales, Juegos y Juguetes, Ciudad y Casa

\begin{tabular}{lcllllll}
\hline \multicolumn{2}{c}{ Animales } & \multicolumn{2}{c}{$\begin{array}{c}\text { Juegos y } \\
\text { Juguetes }\end{array}$} & \multicolumn{2}{c}{ Ciudad } & \multicolumn{2}{c}{ Casa } \\
\hline Vocablo & IDL & Vocablo & IDL & Vocablo & IDL & Vocablo & IDL \\
\hline León & 0.68 & Auto & 0.35 & Casa & 0.69 & Cama & 0.48 \\
Perro & 0.56 & Muñeca & 0.20 & Edificio & 0.55 & Mesa & 045 \\
Gato & 0.49 & Escondida & 0.14 & Auto & 0.49 & Sillón & 0.34 \\
Elefante & 0.37 & Pelota & 0.13 & Colegio & 0.32 & Silla & 0.31 \\
Jirafa & 0.37 & Pista & 0.12 & Árbol & 0.23 & Tele & 0.31 \\
Tigre & 0.36 & Max-steel & 0.11 & Calle & 0.23 & Mueble & 0.30 \\
Mono & 0.21 & Barbie & 0.10 & Persona & 0.21 & Cocina & 0.28 \\
Pez & 0.18 & A u to-c . & 0.09 & Mesa & 0.10 & Pieza & 0.28 \\
& & remoto & & & & & \\
Caballo & 0.18 & & - & Tienda & 0.10 & Baño & 0.18 \\
Cebra & 0.18 & & - & & - & Puerta & 0.17 \\
Tiburón & 0.17 & & - & & - & Ventana & 0.16 \\
Conejo & 0.13 & & - & & - & & - \\
Oreja & 0.17 & Chala & 0.10 & Papa-frita & 0.15 & & - \\
Codo & 0.14 & & - & Lenteja & 0.13 & & - \\
Muñeca & 0.12 & & - & Puré & 0.13 & & - \\
Hueso & 0.10 & & - & & - & & - \\
\hline & & & & & & & \\
\hline
\end{tabular}

El centro "ciudad" presentó problemas para los alumnos. Incluso la unidad "auto" fue también considerada en el campo "medios de transportes". El centro resultó complejo debido a que era extenso en cuanto a las menciones que los estudiantes podían elegir.

Cama, mesa, silla, sillón y tele son las palabras más recordadas en la muestra, pese a que los índices de disponibilidad léxica son bastante pobres, como se muestra en la tabla 3. Es posible que los niños y niñas realizaran este tipo de elección debido a que son los lugares que más utilizan en sus propios hogares, en desmedro de otras unidades. 
"Profesiones y oficios" resultó ser un centro complejo para los estudiantes, con índice muy por debajo de la media para la muestra, más aún considerando que los estudiantes tampoco tenían algunos conceptos integrados; por ejemplo: reparador de casa (0.002), persona del circo (0.003) o atrapar-ladrón (0.008). Esto puede ser observado en la tabla 4.

\section{Tabla 4}

\section{Índice de Disponibilidad Léxica para los Centros Profesiones y Oficios, Escuela y Colores}

\begin{tabular}{lclllc}
\hline Profesiones y Oficios & \multicolumn{2}{c}{ Escuela } & \multicolumn{2}{c}{ Colores } \\
\hline Vocablo & IDL & Vocablo & IDL & Vocablo & IDL \\
\hline Profesor & 0.28 & Mesa & 0.61 & Rojo & 0.73 \\
Profesora & 0.20 & Silla & 0.60 & Amarillo & 0.62 \\
Doctor & 0.18 & Pizarra & 0.46 & Verde & 0.51 \\
Bombero & 0.17 & Sala & 0.32 & Azul & 0.46 \\
Vendedor & 0.12 & Patio & 0.23 & Negro & 0.39 \\
Carabinero & 0.12 & Ventana & 0.14 & Morado & 0.38 \\
Constructor & 0.11 & Cuaderno & 0.14 & Café & 0.36 \\
Policía & 0.10 & Puerta & 0.13 & Blanco & 0.35 \\
Enfermera & 0.09 & Niño & 0.12 & Rosado & 0.35 \\
Mecánico & 0.08 & Libro & 0.12 & Celeste & 0.24 \\
Futbolista & 0.07 & Lápiz & 0.11 & Piel & 0.22 \\
Conejo & 0.13 & & - & & - \\
Oreja & 0.17 & Chala & 0.10 & Papa-frita & 0.15 \\
Codo & 0.14 & & - & Lenteja & 0.13 \\
Muñeca & 0.12 & & - & Puré & 0.13 \\
Hueso & 0.10 & & - & & - \\
\hline
\end{tabular}

El centro "escuela", aún cuando comparte algunas unidades, logra tener respuestas un poco más cohesionadas; sin embargo, hay varias repeticiones (e.g, mesa, silla), lo que podría indicar la necesidad de revisar los centros de interés presentados a los niños y niñas, para evitar que las evocaciones léxicas se solapen en las elecciones de las unidades léxicas. 
Por último, el centro "colores" posee los índices más altos de la muestra y los menos dispersos, tal como se visualiza en la tabla 4. Llama la atención, por ejemplo, la aparición del color piel y que puede ser el reflejo de la influencia de la televisión en los estudiantes, toda vez que esta mención está presente en todos los canales de televisión por cable.

\section{Conclusiones}

De acuerdo al estudio realizado se pueden desprender las siguientes conclusiones. En términos generales, se debe considerar que los desempeños de todos los alumnos de 1er año básico evaluados tienen un repertorio más disperso al exhibir índices cercanos a 0 , mostrando que el promedio de sus resultados no sobrepasa el índice 0.15, con excepción de los colores, que tiene 0.25. Además, el mayor número de palabras diferentes evocadas por los estudiantes se dio en los centros de interés correspondientes a "bebidas y alimentos", "ciudad" y "casa". Se desprende de estos resultados que el índice de disponibilidad léxica no necesariamente equivale a la mayor cantidad de palabras emitidas, tal como se observa en este estudio, donde los niños y niñas mostraron un mayor índice de disponibilidad en el centro de interés los "colores" pero un mayor número de vocablos emitidos en el centro de interés "bebidas y alimentos"; "ciudad" y "casa".

En relación al promedio de palabras evocadas por los estudiantes, se observa que el promedio más alto obtenido alcanza las 12 palabras en el centro de interés "bebidas y alimentos"; "animales" y "casa". En este sentido, en el contexto nacional no se cuenta con un estándar que dé cuenta de la cantidad esperada de vocablos que los niños y niñas de 6 y 7 años deberían producir.

Respecto a la hipótesis de trabajo, queda de manifiesto que existen diferencias entre los estudiantes encuestados. Las niñas presentan un mayor grado de cohesión y en todos los campos elíndice de disponibilidad léxica es mayor que el de los niños. Esta situación está directamente relacionada con la teoría (Gillani, 2010) que índica que las niñas aprenden de manera más temprana el lenguaje que los niños. Sin embargo, si se revisan los otros índices investigados, "promedio de palabras" y "número de vocablos", la realidad es distinta por cuanto las diferencias entre niños y niñas en el primer caso son mínimas, mientras que en el segundo los varones son capaces de evocar un mayor número de unidades. 
Los vocablos más disponibles en los estudiantes son sustantivos con representaciones concretas y cercanas a la experiencia de los niños y niñas, esto además se vincula con los centros de interés seleccionados para este estudio, pues todos ellos estimulan prioritariamente la producción de vocabulario con estas características. En cuanto a la complejidad fonológica y metría de las palabras más evocadas por los estudiantes, se observa variedad en su composición, lo cual se ajusta a la etapa de desarrollo lingüístico que los niños y niñas de 6 y 7 años deberían presentar. A esta edad el repertorio fonológico debería estar adquirido en su totalidad.

El conocer cuáles son las palabras disponibles en los niños y niñas de 6 y 7 años, puede constituirse en un aspecto valioso a considerar para la evaluación y la enseñanza del lenguaje oral y escrito. En relación a la evaluación del lenguaje, el conocimiento del índice de disponibilidad léxica esperado para las diferentes edades, puede ayudar a identificar tempranamente dificultades en el nivel semántico del lenguaje y puede actuar como predictor de dificultades del proceso de lectura y escritura. Concebir el índice de disponibilidad léxica como un referente que guíe la instrucción explícita y sistemática, permite plantear metas de desarrollo del lenguaje oral y escrito al servicio de la enseñanza.

Por lo tanto, los índices observados pueden interpretarse como una necesidad de reformular la enseñanza de la lengua materna y el trabajo de la expansión del vocabulario, para que estos sean impartidos de manera explícita y sistemática. El desarrollar el elemento léxico en los estudiantes es fundamental y debiese trabajarse durante todo el proceso académico de los estudiantes. En este sentido se señala que una enseñanza explícita del léxico potencia el incremento de vocabulario y por ende de las habilidades lingüísticas vinculadas a los procesos de lectura, escritura y todos los aprendizajes académicos en general (e.g, Borrero, 2008; Cuetos, 2009; Graves, 2009; Landauer \& Dumais, 1997).

Algunas de las dificultades que presentaron los estudiantes en relación con algunos centros de interés se asocian al no contar con una palabra para designar las cosas, teniendo que recurrir a la descripción de la misma; por ejemplo: "señor que maneja un camión", en reemplazo de "chofer".

Respecto de los resultados, es necesario indagar más acerca de la influencia que tiene la televisión en los contextos comunicativos de los 
estudiantes encuestados, puesto que la irrupción de algunos términos como "color piel" o de unidades léxicas tomadas del inglés se puede deber justamente a la exposición a programas en español neutro o donde hay predominio, sobre todo de nombres en inglés, de personajes de televisión o de tecnologías de la comunicación con las que tienen contacto. Respecto de esto mismo, podemos considerar un antecedente de facto observado en chilenos que se vieron expuestos a programas mexicanos en las décadas 70' y 80', principalmente novelas y los programas de Chespirito, quienes adoptaron giros lingüísticos en el habla cotidiana e incluso algunas unidades de origen Nahual, como "chapulín", por ejemplo.

Finalmente, es necesario reafirmar la idea de que el desarrollo del componente léxico es preponderante para tareas de comprensión lectora y de escritura (e.g, Bartol, 1997; Galloso, 2004) tareas que no se pueden realizar en propiedad si los estudiantes tienen un léxico pasivo que es básico y uno activo que es restringido. Realizar este trabajo de manera permanente en el tiempo quizás permita concretar el anhelo de tener alumnos con conciencia crítica y hábiles comunicadores en el contexto oral y escrito. 


\section{Referencias bibliográficas}

Allen, V. (1984). Techniques in teaching vocabulary. Oxford: Oxford University Press.

Anderson, R. C., \& Freebody, P. (1981). Vocabulary knowledge. In J. T. Guthrie (Ed.), Comprehension and teaching: Research reviews (pp. 77-117). Newark, DE: International Reading Association.

Baumann, J. F., Kame'Enui, E. J., \& Ash, G. E. (2003). Research on vocabulary instruction: Voltaire redux. In J. Flood, D. Lapp, J. R. Squire, \& J. M. Jensen (Eds.), Handbook of Research on Teaching the English Language Arts, Second Edition (pp. 752-785). Mahwah, NJ: Lawrence Erlbaum.

Bartol, J. (2006). Disponibilidad léxica y selección del vocabulario. Revista Española de Lingüística, 36, 379-396.

Borrero, L. (2008). Enseñando a leer: Teoría, práctica e intervención. Colombia: Norma

Bruning, R., Schorow, S. Norby, N. \& Ronning, R. (2005). Psicología cognitiva y de la instrucción. Madrid: Pearson Educación S.A.

Cuetos, F. (2009). Psicología de la lectura. Barcelona: Wolters Kluwer Educación.

Educar Chile. (2012). Efemeride. 11 de mayo de 1904: Nace Salvador Dalí. Recuperado de www.educarchile.cl/Portal.Base/ Web / VerContenido.aspx?ID0=214482

Elleman, A. M., Lindo, E. J., Morphy, P., \& Compton, D. L. (2009). The impact of vocabulary instruction on passagelevel comprehension of school-age children: A meta-analysis. Journal of Research on Educational Effectiveness, 2, 1-44.

Eyzaguirre, B. y Fontaire, L. (2008). Las escuelas que tenemos. Santiago: Centro de estudios públicos. 
Ferreira, R. y Echeverría, M. (2010). Redes semánticas en el léxico disponible de inglés L1 e inglés LE. Onomázein, 21, 133-153.

Gairns, R. \& Redman, S. (1986). Working with words. A guide to teaching and learning vocabulary. Cambridge: Cambridge University Press.

Galloso, M. (2004). Déficit léxico y comprensión lectora. Puertas a la Lectura, 17, 35-38.

Gillani, E. (2010). El factor género en el proceso de adquisición de lenguas: revisión crítica de los estudios interdisciplinares. Recuperado de www.linred.es/articulos_pdf/LR_ articulo_30042010.pdf?

Lin. (2012). El estudio de disponibilidad léxica de los estudiantes chinos de español como lengua extranjera. Marcoele Revista de Didáctica, 14, 1-14.

Lockett, J., \& Shore, W. (2003). A narwhal is an animal: Partial word knowledge biases adults' decisions. Journal of Psycholinguistic Research, 32 (4), 477-496.

López, J. \& Strassburger, C. (1987). Otro cálculo del índice de disponibilidad léxica. Presente y perspectiva de la investigación computacional en México. Actas del simposio de la asociación mexicana de lingüística aplicada.

Morgan, J. \& Rinvolucri, M. (1986). Vocabulary. Oxford: Oxford University Press.

Nation, I (1990). Teaching and learning vocabulary. Rowely (Mass.): Newbury House.

National Reading Panel (2000). Report of the National Reading Panel: Teaching Children to Read: An Evidence-based Assessment of the Scientific Research Literature on Reading and its Implications for Reading Instruction. Washington, DC: National Institute of Child Health and Human Development. 
Palapanidi, K. (2012). La aplicación de la disponibilidad léxica a la didáctica del léxico de LE. Revista Nebrija de Lingüística Aplicada 11 (6), 69-77.

Perfetti, C. A. (1985). Reading ability. New York: Oxford University Press.

Rosenshine, B. (1987). Explicit teaching and teacher training. Journal of Teacher Education, 38 (3), 34-36.

MINEDUC. (2012). Recuperado de http: / / www.mineduc.cl/ contenido_ int.php?id_contenido=23428\&id_portal $=1 \& i d$ seccion $=4532$

Stahl \& Fairbanks, (1986). The effects of vocabulary instruction: A modelbased data analysis. Review of Educational research, $56,72-110$

Shefelbine, J. (1990). Student factors related to variability in learning word meanings from context. Journal of Reading Behavior, 22 (1), 71-97.

Shore, W. \& Kempe, V. (1999). The role of sentence context in accessing partial knowledge of word meanings. Journal of Psycholinguistic Research, 28 (2), 145-163.

Urzúa, P;Saéz, K; Echeverría, M. (2006). Disponibilidad léxica matemática. Análisis cuantitativo y cualitativo. RLA. Revista de Lingüística Teórica y Aplicada, 44 (2), 59-76

Valencia, A. (1997). Disponibilidad léxica. Muestreos y estadísticos. Onomazein, 2, 197-226. 
